\title{
Alteration of Lipid Peroxide and Endogenous Antioxidant Contents in Retina of Streptozotocin-Induced Diabetic Rats: Effect of Vitamin A Administration
}

\author{
Chihiro NISHIMURA and Kinya KURIYAMA \\ Department of Pharmacology. Kyoto Prefectural University of Medicine. \\ Kawaramachi-Hirokoji, Kamikyo-ku, Kyoto 602, Japan
}

Accepted January 7, 1985

\begin{abstract}
Possible involvement of lipid peroxide (LPO) in the occurrence of diabetic retinal lesion was investigated using streptozotocin-induced diabetic rats. Young male Wistar rats weighing 100-150 g were made diabetic by daily intraperitoneal injection of $30 \mathrm{mg} / \mathrm{kg}$ streptozotocin (STZ) for 5 days. Five weeks after the termination of STZ-treatment, when animals maintained typical hyperglycemia, the tissue level of LPO, estimated by the thiobarbituric acid method in the presence of $0.5 \mathrm{mM}$ EDTA, was found to be augmented in the kidney. At 7 to 9 weeks after the STZ-treatment, the content of LPO in the retina also exhibited a significant increase, while those in the serum, brain and peripheral nerves showed no alteration. This increment of LPO in the kidney and retina was accompanied by the concomitant reduction of fat-soluble antioxidants determined by the ferric chloride-bipyridyl reaction, and insulin treatment $(10 \mathrm{u} / \mathrm{rat} / \mathrm{day}$, s.c.) completely eliminated the increased formation of LPO in these organs. When diabetic rats were treated with retinol acetate, which had an inhibitory effect on LPO formation in retinal homogenate, the increase in LPO content was found to be significantly suppressed, especially in the retina. These results suggest that the STZ-induced diabetic state may elicit an increased formation of LPO in the retina and kidney, both of which are known to be main organs having typical diabetic lesions.
\end{abstract}

Over the past decade, a considerable body of evidence has been accumulated which indicates that peroxidation of membrane lipids may lead to an irreversible impairment of cellular integrity. Lipid peroxidation is well known to be the consequence of oxidative deterioration of polyunsaturated lipids, a process in which free radicals play an intermediate role.

Since the retina has a rich supply of oxygen and contains a large quantity of polyunsaturated fatty acids, especially in the photoreceptor layer, it is highly conceivable that retinal tissue is susceptible to oxidative damages which lead to lipid peroxidation. In fact, clinical studies have shown that exposure of premature infants to high levels of oxygen causes the destruction of retinal vascular systems (1). Experimental studies have revealed that the level of lipid peroxide (LPO) in retinal tissue is increased in animals exposed to high concentrations of oxygen (2. 3). Retinal damages induced by irradiation (4), light (5) and iron (6) have also been reported to be dependent upon lipid peroxidation elicited by free radical formation.

On the other hand, a wealth of data has been collected on the pathology of diabetic retinopathy. This complication, together with diabetic nephropathy, exhibits a typical vascular lesion called microangiopathy which is known to be one of the characteristic manifestations in diabetes. Although diabetic retinopathy has now become a major cause of new blindness in adulthood, the exact pathological mechanism as well as how to prevent the progress of this lesion still remain to be elucidated. Since serum LPO level was 
reported to be increased in diabetic patients (7), it appears relevant to study whether or not lipid peroxidation is involved in the pathogenesis of various diabetic complications. In this study, we have examined the alteration of LPO level in experimentally diabetic animals and the possible role of endogenous antioxidants in protection against peroxidative injury in various organs.

\section{Materials and Methods}

Handling of animals: Male Wistar rats weighing 100-150 g were made diabetic by daily intraperitoneal injection of streptozotocin (STZ) for 5 days with free access to laboratory chow and water. Glucose level in serum collected from decapitated animals was measured by an enzymatic method using glucose oxidase (8). In insulin replacement studies, insulin zinc suspension (insulin Novo Lente; $10 \mathrm{IU} /$ rat, s.c.) was administered daily to diabetic rats at 4 to 5 weeks after the injection of STZ until the day of experiment. The total vitamin A contained in laboratory chow was $1.100 \quad \mathrm{U} / 100 \mathrm{~g}$ diet, and this amount was found to be sufficient for the daily requirement in adult rodents. Supplemental vitamin A (retinol acetate: 1,000 $\mathrm{IU} /$ rat, p.o.) was administered twice a week to animals at 4 to 5 weeks after STZ treatment until the day of sacrifice, which may double the intake of vitamin A.

Determination of LPO: LPO in serum (9) and tissue (10) was estimated by the thiobarbituric acid (TBA) reaction in the presence of $0.5 \mathrm{mM}$ ethylenediaminetetraacetic acid (EDTA) to inhibit non-enzymatic lipid peroxidation during the manipulation (11). and it was expressed in terms of pmoles or nmoles malondialdehyde (MDA) present in the assay. Fifty $\mu l$ of $10 \%(\mathrm{~W} / \mathrm{V})$ tissue suspension containing $1 \mathrm{mM}$ of EDTA was taken for the following fluorometric determination (excitation: $515 \mathrm{~nm}$, emission: 553 $\mathrm{nm}$ ) of LPO using tetraethoxypropane as a standard.

Determination of antioxidants: Watersoluble antioxidant was measured by the use of the stable free radical 1,1-dipheny!-2picrylhydrazyl (DPPH) in the supernatant fraction prepared by $5 \%$ trichloroacetic acid (TCA) precipitation of tissue homogenates
(12, 13). The amount is expressed in terms of $n$ equiv. using ascorbic acid as a standard. Lipid-soluble antioxidant in the chloroformmethanol $(2: 1)$ extract of the former TCA precipitate was determined by ferric chloridedipyridyl reaction. which was expressed in terms of $n$ equiv. using $\alpha$-tocopherol as a standard (13).

Chemicals used: Streptozotocin and retinol acetate (Type III) were purchased from the Sigma Chemical Co.; and 1,1-diphenyl-2picrylhydrazyl, ascorbic acid, $\alpha, \alpha^{\prime}$-dipyridyl and $\alpha$-tocopherol were obtained from Nakarai Chemicals, Ltd. Thiobarbituric acid and 1,1,3,3-tetraethoxypropane were obtained from Tokyo Kasei Kogyo Co., Ltd., and insulin Novo Lente ${ }^{\mathbb{R}}$ MC was from Novo Industry $\mathrm{A} / \mathrm{S}$.

\section{Results}

Effect of STZ on body weight and serum level of glucose: Single intraperitoneal injection of STZ $(70 \mathrm{mg} / \mathrm{kg})$ to rats elicited an increase in serum level of glucose at $4 \mathrm{hr}$ after the injection. which was followed by slight and transient hypoglycemia at $7 \mathrm{hr}$ and subsequent remarkable hyperglycemia at 24 hr after the injection as shown in Fig. 1.

Several weeks after the STZ-treatment, however, some of the rats were found to recover normoglycemia. Therefore, repeated intraperitoneal injection of STZ was applied so as to make a chronically hyperglycemic animal as a diabetic model.

When young male Wistar rats weighing $100-150 \mathrm{~g}$ were treated with $15 \mathrm{mg} / \mathrm{kg}$ of STZ daily for 5 days, they exhibited a mild elevation of serum glucose and a normal pattern of weight gain (Fig. 2). On the other hand, the treatment with $30 \mathrm{mg} / \mathrm{kg}$ of STZ induced a marked hyperglycemia (400-600 $\mathrm{mg} / \mathrm{dl}$ ) as well as the suppression of a gain in body weight.

LPO content in various organs and effect of insulin administration: Determination of LPO content in various organs of hyperglycemic rats revealed that the tissue level of LPO in kidney was significantly increased at 5 weeks after the STZ-treatment (Fig. 3). Though single administration of STZ had no notable effect on retinal level of LPO (Fig. 1). a significant increment of LPO in the retina 

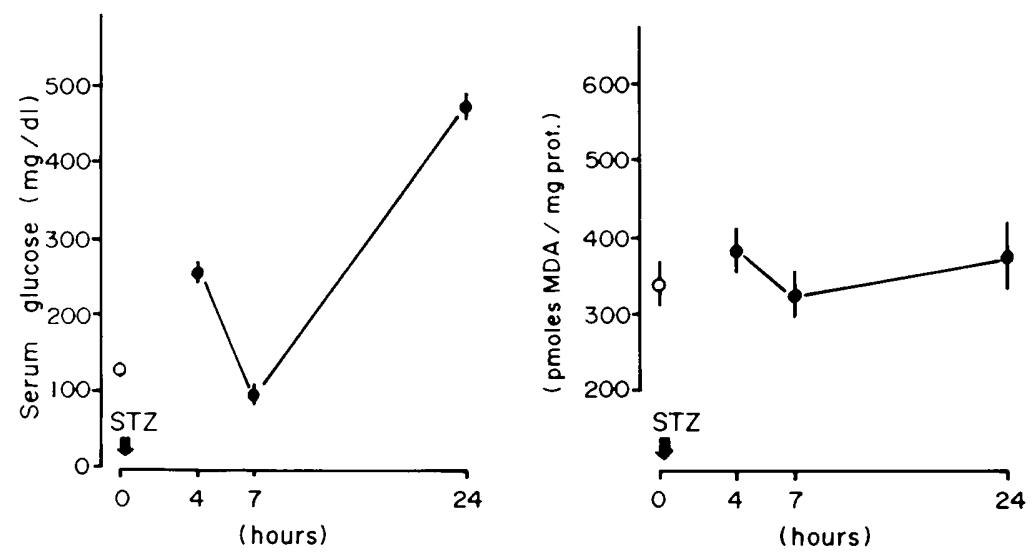

Fig. 1. Effect of single injection of streptozotocin (STZ) on serum level of glucose (left) and lipid peroxide content (right) in rat retina. STZ $(70 \mathrm{mg} / \mathrm{kg})$ was intraperitoneally injected to male Wistar rats fasted for $15-16 \mathrm{hr}$. Each point represents the mean \pm S.E.M. obtained from 6 animals.
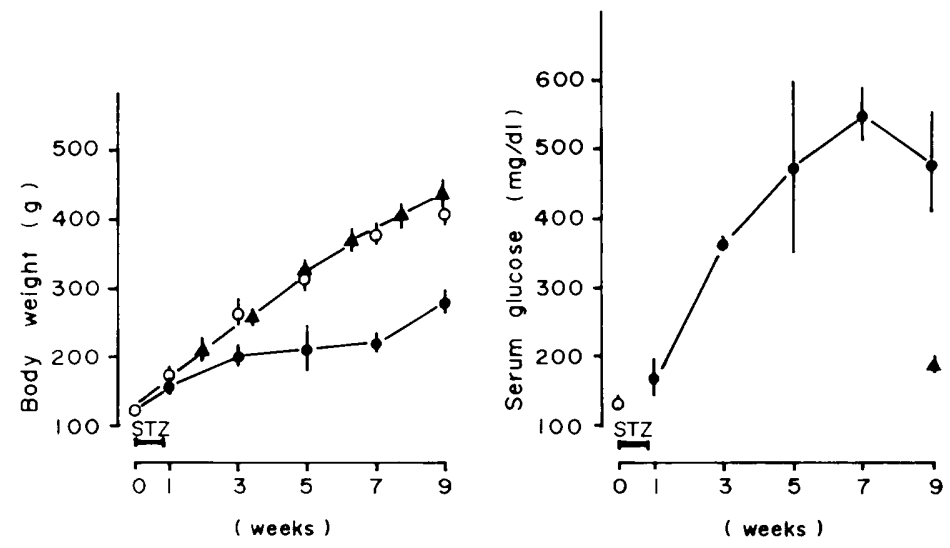

- Control

- STZ: $15 \mathrm{mg} / \mathrm{kg} \times 5$, i.p.

- STZ: $30 \mathrm{mg} / \mathrm{kg} \times 5$, l.p.

Fig. 2. Effect of repeated treatment with STZ on body weight and serum level of glucose in rats. STZ was intraperitoneally injected to male Wistar rats once a day for 5 days. Each point represents the mean \pm S.E.M. obtained from 3 to 15 animals.

was observed at 7 to 9 weeks after the repeated injection of STZ (Fig. 4). The level of LPO in serum as well as other nervous tissues including whole brain, spinal cord and sciatic nerve, as presented in Fig. 3, exhibited no alteration, which indicates the specificity of increased LPO formations in the kidney and retina in these diabetic animals.

On the other hand, daily subcutaneous administration of insulin effectively suppressed the elevation of serum level of glucose at 9 weeks after STZ-treatment, while the increased formation of LPO in the retina and kidney was found to be completely eliminated by this treatment (Fig. 5). Thus the alteration of LPO in these organs induced by STZ-treatment appears to be dependent upon continuous elevation of the serum level of glucose.

Content of endogenous antioxidants: Though the level of water-soluble antioxidant, endogenously contained in tissue and serum, was not influenced by STZ-treatment (Fig. $6)$, the determination of lipid-soluble anti- 

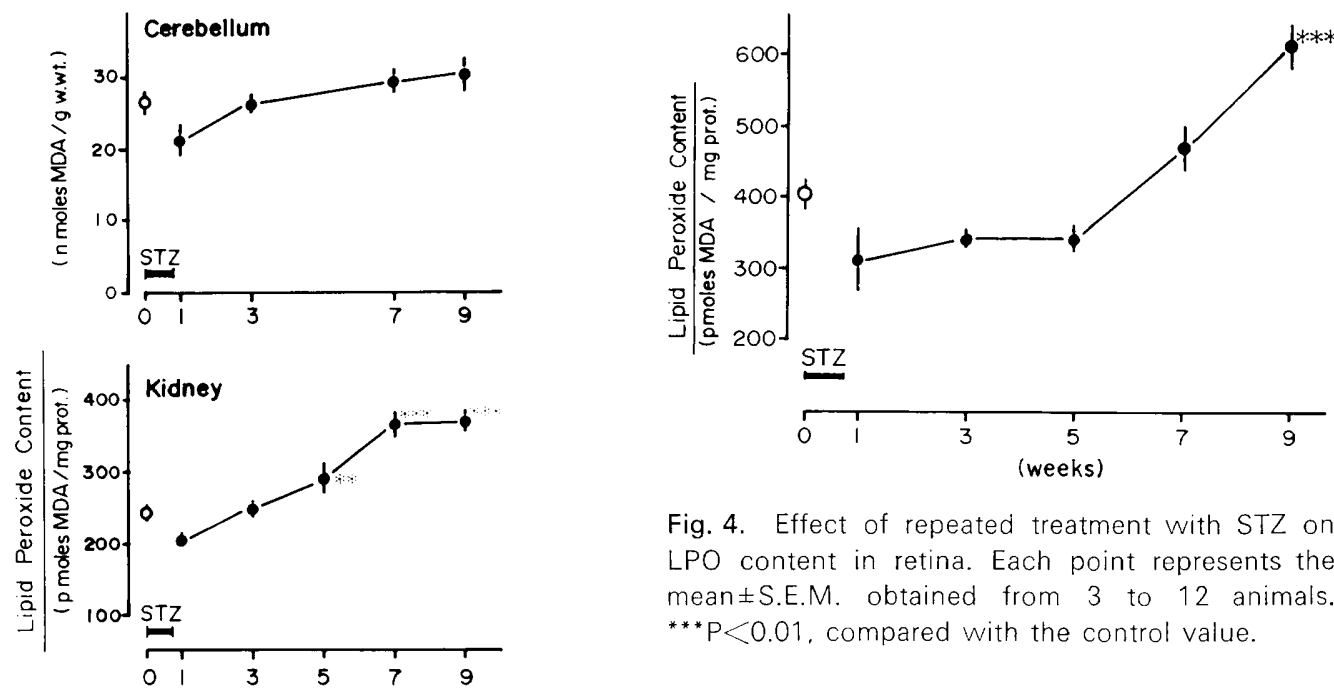

Fig. 4. Effect of repeated treatment with STZ on LPO content in retina. Each point represents the mean \pm S.E.M. obtained from 3 to 12 animals. ${ }^{* * *} \mathrm{P}<0.01$, compared with the control value.

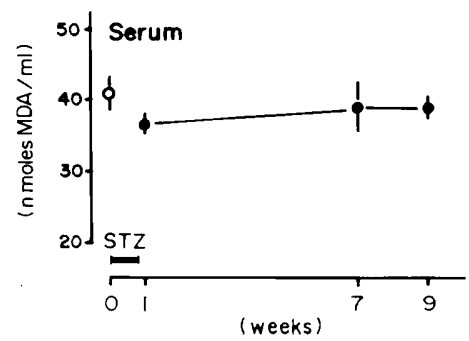

acid remarkably accelerated LPO formation as reported previously (14). On the other hand, $\alpha$-tocopherol, retinol and retinoic acid, which are known to be endogenous lipophilic antioxidants, significantly suppressed the formation of LPO in retinal homogenate.

Retinoids are documented to play an essential role in visual function, but their antioxidative action in the retina has not drawn much attention. Thus, the effect of in vivo administration of retinol acetate on LPO content was further examined in the rats treated with STZ. As shown in Fig. 9, STZinduced increment of LPO content in the retina was found to be significantly attenuated by retinol administration. The increment of

oxidant revealed that the content was specifically attenuated in the retina and kidney (Fig. 7).

Effect of retinol acetate administration: When retinal homogenate was incubated with $10^{-4} \mathrm{M}$ of ferric chloride at $37^{\circ} \mathrm{C}$ in an aerobic environment, time-dependent formation of LPO in the homogenate was observed. As shown in Fig. 8, addition of the antioxidant enzyme superoxide dismutase $(250 \mathrm{u} / \mathrm{ml})$ had no effect on the lipid peroxidation in the homogenate. Hydrophilic antioxidants such as 1.2-dihydroxybenzene3,5-disulfonic acid disodium salt (tiron). glutathione, and mannitol at the concentration of $10^{-4} \mathrm{M}$ showed no preventive action on the peroxidation, while $L$-ascorbic LPO content in the kidney of diabetic rats was, however, not affected by this treatment, which indicates the potent protective action of the latter retinoid in the visual organ.

\section{Discussion}

Early triphasic blood-sugar response caused by a single intraperitoneal injection of STZ coincided with the former report by Junod et al. (15). Since the content of LPO in retina was not affected by this transient fluctuation of serum level of glucose, and daily administration of insulin completely eliminated the alteration of LPO level in the kidney and retina, it is reasonable to assume that the continuous hyperglycemic state induced by STZ may cause an augmented 

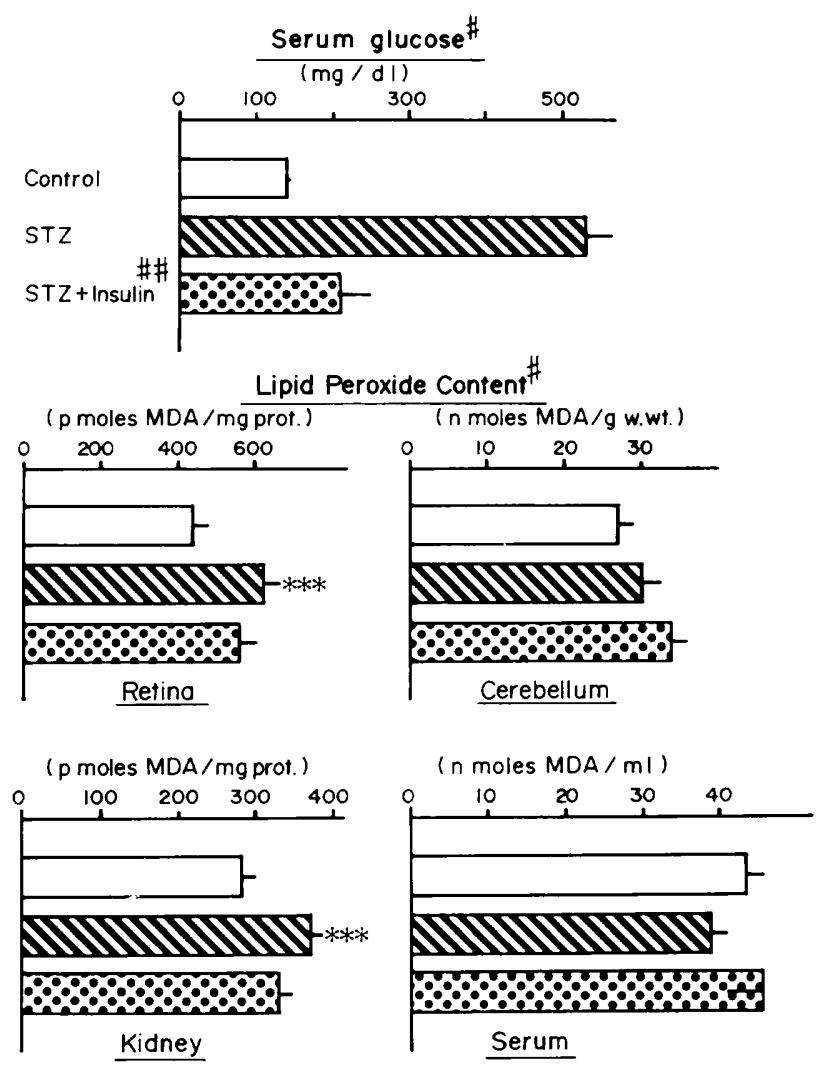

Fig. 5. Effect of insulin-treatment on the alteration of LPO content following STZ-induced hyperglycemia. Each bar represents the mean \pm S.E.M. obtained from 6 to 15 animals. "Determined at 9 weeks after SZT-treatment. "\#\# Administered subcutaneously (10 $\mathrm{u} / \mathrm{rat} / \mathrm{day})$ during $4.5-9$ th week after STZtreatment. $\quad{ }^{* *} \mathrm{P}<0.01$, compared with the corresponding control value.
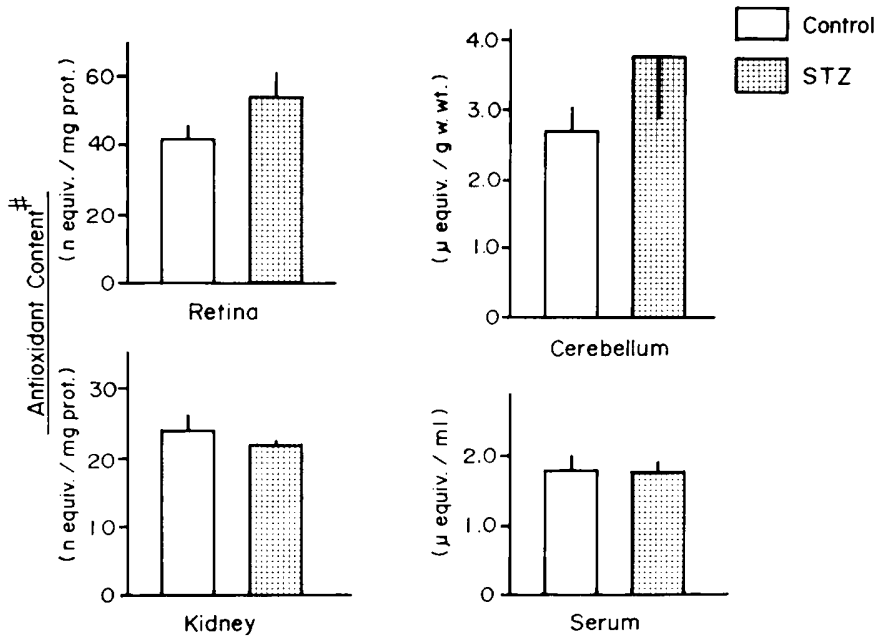

Fig. 6. Effect of repeated treatment with STZ on content of water-soluble antioxidants in retina. cerebel!um, kidney and serum. Each bar represents the mean \pm S.E.M. obtained from 7 to 9 animals. \#Determined at 9 weeks after STZ-treatment. 

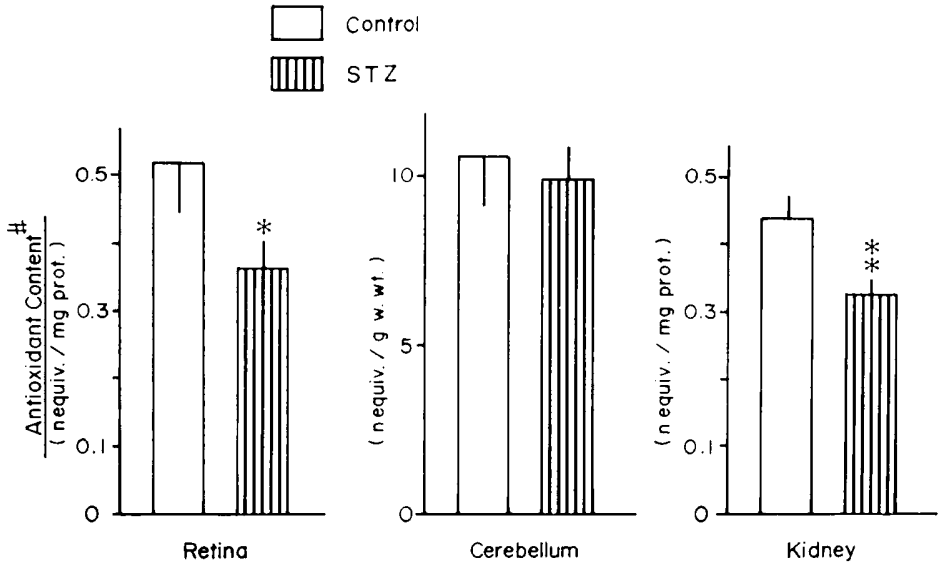

Fig. 7. Effect of repeated treatment with STZ on content of lipid-soluble antioxidants in retina, cerebellum and kidney. Each bar represents the mean \pm S.E.M. obtained from 7 to 10 animals. \#Determined at 9 weeks after STZ-treatment. ${ }^{*} \mathrm{P}<0.05$ or ${ }^{*} \mathrm{P}<0.02$, compared with the corresponding control value.

formation of LPO in these organs.

It is well known that vascular lesion, especially microangiopathy, is one of the serious complications in diabetic patients. A histological feature common to this vascular disorder is reported to be a thicker yet more porous basement membrane structure throughout the microvasculature (16). Considering the fact that both the retina and kidney are the main organs having this type of complications, it seems highly likely that there may exist some interrelation between the increased formation of LPO and the occurrence of pathological lesions in these organs. Furthermore, lipid peroxidation per se had been demonstrated to play an important role in retinal degeneration induced by various injurious factors (1-6). Actually, direct intravitreal injection of LPO has been found to elicit progressive and irreversible attenuation of the amplitude of ERG, indicating functional deterioration of retina (17). Thus, the increment of LPO content observed in the retina of STZ-induced diabetic rat may be indicative of retinal dysfunction associated with diabetic retinopathy, as reported previously in human subjects (18). Murata et al. (14) reported that the rate of lipid peroxidation in a retinal homogenate preparation was accelerated in alloxan-induced diabetic rats. This result has an essential agreement with the results obtained in this study that the endogenous level of LPO in the retina is increased following the continuous hyperglycemia induced by STZ administration.

Several protective mechanisms against lipid peroxidation have been shown to exist in the eye, among which superoxide dismutase (19) and $\alpha$-tocopherol (20) are now considered to be a major first line of defense in such mechanisms. Under certain pathological conditions, however, oxidative mechanisms may exceed the ability of retinal tissue to protect itself, and this may account for our present finding that endogenous level of lipid soluble antioxidant was significantly attenuated in accordance with the simultaneous augumentation of LPO content in the retina and kidney. When retinal homogenate containing ferric ion incubated with various antioxidative substances, lipid-soluble antioxidants specifically suppressed the generation of LPO in the homogenate, while superoxide dismutase and other water-soluble antioxidants did not show any significant inhibitory effect on lipid peroxidation.

$\alpha$-Tocopherol, a lipophilic vitamin, has been shown to prevent the oxidative damage in rod outer segments, but it was found that the endogenous level of tocopherol was insufficient to eliminate the peroxidation of membranous lipid completely (20). These results suggest the existence of other potent protective mechanisms against peroxidation in the retina. In fact, our present investigation 


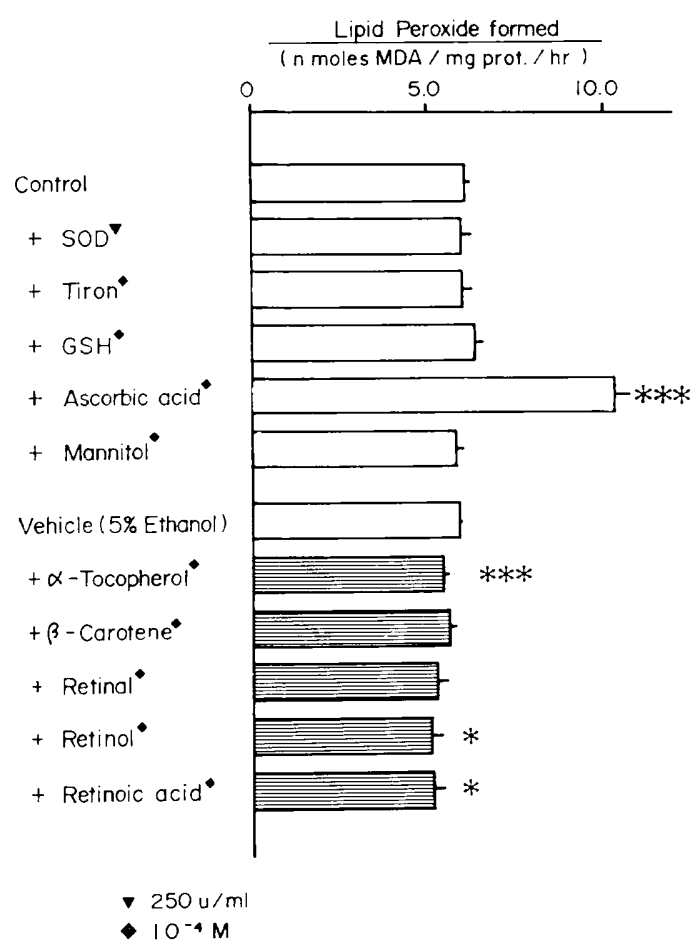

Fig. 8. Effect of various antioxidants on ferric ioninduced lipid peroxide formation in retinal homogenate. Following the addition of various antioxidants to retinal homogenate including $10^{-4}$ $M$ of ferric chloride, each sample was incubated at $37^{\circ} \mathrm{C}$ for $60 \mathrm{~min}$. Lipid peroxidation during the incubation was estimated by subtracting the endogenous level of LPO present in the original tissue preparation from each experimental value. Each bar represents the mean \pm S.E.M. obtained from 3 to 6 experimenta. ${ }^{*} \mathrm{P}<0.05$ or ${ }^{* *} \mathrm{P}<0.01$. compared with the corresponding control value.

has indicated that retinol exhibits antilipoperoxidative effects in the retina both in vitro and in vivo. Antioxidative actions of retinol have already been demonstrated in subcellular fractions from the liver, kidney and spleen of normal rats (21). Although large doses of retinoids are known to be toxic to biological membranes $(22,23)$, it has been shown that these compounds prevent oncogenic transformation induced by chemicals (24) or $r$-ray irradiation (25), which recently has attracted the attention of many scientists. While the exact mechanism of this inhibitory effect of retinoids on carcinogenesis
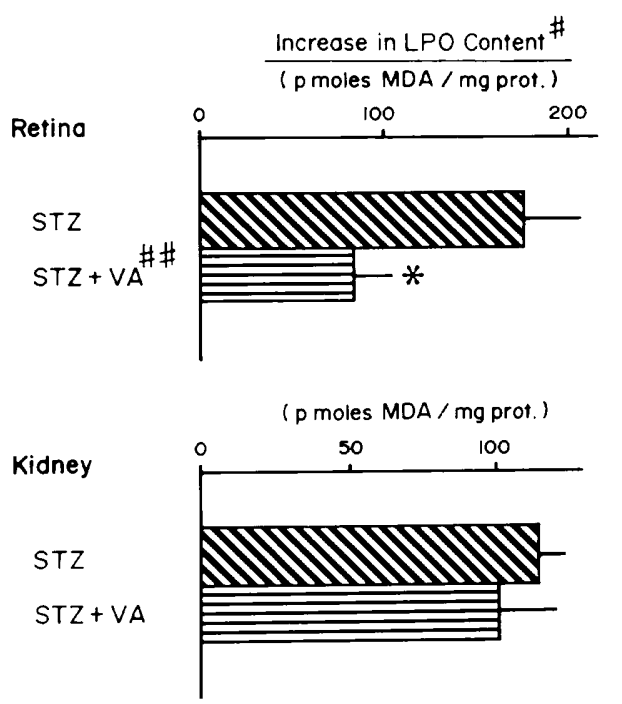

Fig. 9. Effect of retinol acetate (VA) administration on STZ-induced increase in LPO content in retina and kidney. Each bar represents the mean \pm S.E.M. obtained from 8 to 11 animals. "Determined at 9 weeks after STZ-treatment, when LPO contents in control retina and kidney were $437.6 \pm 29.8$ (11) and $274.8 \pm 12.7$ (11) pmoles $\mathrm{MDA} / \mathrm{mg}$ prot., respectively. "\#\#Administered twice a week $(1,000$ IU/rat, p.o.) during 4.5-9th week after STZtreatment. ${ }^{*} \mathrm{P}<0.05$, compared with the corresponding control value.

still remains to be determined, it has been proposed that the quenching reaction of these compounds with singlet oxygen which leads to peroxidation may take part in their anticancer effect (26). In view of these studies and present findings, it seems permissible to assume that retinoids may be involved in physiological protective mechanisms against lipid peroxidation in the retina in addition to their photo-receptive functions as visual pigment. Therefore, it may be pharmacologically significant to administer this vitamin or its synthetic analogs so as to prevent the excessive formation of LPO such as found in the retina of diabetic rats. Further studies on the exact site as well as the specificity of this antiperoxidative action of vitamin $A$ in the retina, however, may be required in order to bring this idea into clinical practice.

Acknowledgement: This work was supported in 
part by a Grant in Aid (No, 58770172, 1983) from the Ministry of Education. Science and Culture of Japan.

\section{References}

1 Patz, A.: Current status of the role of oxygen in retrolental fibroplasia. Invest. Ophthalmol. Vis. Sci. 15, 337-339 (1976)

2 Hiramatsu, T.Y., Hirato, K., Nishigaki, I. and Yagi, K.: The formation of lipoperoxide in the retina of rabbits exposed to high concentration of oxygen. Experientia 32, 622-623 (1976)

3 Yagi, K., Matsuoka, S., Ohkawa, H., Ohishi, N., Takeuchi, Y. and Sakai, H.: Lipoperoxide level of the retina of chick embryo exposed to high concentration of oxygen. Clin. Chim. Acta 80, 355360 (1977)

4 Hiramitsu, T.Y., Majima, Y., Hasegawa, Y. and Hirata, K.: Role of lipid peroxide in the induction of retinopathy by $X$-irradiation. Acta Soc. Ophthalmol. Japon. 78, 819-825 (1974) (Abs. in English)

5 Kagan, V., Shvedova, A., Novikov, K. and Kozlov, Y.: Light-induced free radical oxidation of membrane lipids in photoreceptors of frog retina. Biochim. Biophys. Acta 330, 76-79 (1973)

6 Hiramitsu, T.Y., Majima, Y., Hasegawa, Y., Hirata, K. and Yagi, K.: Lipoperoxide formation in the retina in ocular siderosis. Experientia 32, 1324-1325 (1976)

7 Ueda, J.: Lipoperoxides in diabetic retinopathy. Acta Soc. Ophthalmol. Japon. 73, 1367-1378 (1969) (Abs. in English)

8 Raabo, E. and Terkildsen, T.C.: On the enzymatic determination of blood glucose. Scand. J. Clin. Lab. Invest. 12, 402-407 (1960)

9 Yagi, K.: A simple fluorometric assay for lipoperoxide in blood plasma. Biochem. Med. 15, 212-216 (1976)

10 Ohkawa, H., Ohishi, N. and Yagi, K.: Assay for lipid peroxides in animal tissues by thiobarbituric acid reaction. Anal. Biochem. 95, 351-358 (1979)

11 Wills, E.D. and Wilkinson, A.E.: Release of enzymes from lysosomes by irradiation and the relation of lipid peroxide formation to enzyme release. Biochem. J. 99, 657-666 (1966)

12 Blois, M.S.: Antioxidant determinations by the use of a stable free radical. Nature 181, 1199_
1200 (1958)

13 Glavind, J.: Antioxidants in animal tissue. Acta Chem. Scand. 17, 1635-1640 (1963)

14 Murata, T., Nishida, T., Eto, S. and Mukai, N.: Lipid peroxidation in diabetic rat retina. Metab. Pediatr. Syst. Ophthalmol. 5, 83-87 (1981)

15 Junod, A., Lambert, A.E., Stauffacher, W. and Renold, A.E.: Diabetogenic action of streptozotocin: Relationship of dose to metabolic response. J. Clin. Invest. 48, 2129-2139 (1969)

16 Williamson, J.R. and Kilo, C.: Current status of capillary basement membrane disease in diabetes mellitus. Diabetes 26, 65-75 (1977)

17 Armstrong, D., Hiramitsu, T., Gutteridge, J. and Nilsson, S.E.: Studies on experimentally induced retinal degeneration. 1. Effect of lipid peroxides on electroretiongraphic activity in the albino rabbit. Exp. Eye Res, 35, 157-171 (1982)

18 Yonemura, D., Aoki, T. and Tsuzuki, K.: Electroretinogram in diabetic retinopathy. Arch. Ophthalmol. 68, 49-54 (1962)

19 Hall, M.O. and Hall, D.O.: Superoxide dismutase of bovine and frog rod outer segments. Biochem. Biophys. Res. Commun. 67, 1199-1204 (1975)

20 Farnsworth, C.C. and Dratz, E.A.: Oxidative damage of retinal rod outer segment membrane and the role of vitamine E. Biochim. Biophys. Acta 443, 556-570 (1976)

21 Callari, D. and Billitteri, A.: Retinol and lipid peroxidation. Acta Vitaminol. Enzymol. 30, 3843 (1976)

22 Meeks, R.G., Zaharevitz, D. and Chen, R.F.: Membrane effects of retinoids: Possible correlation with toxicity. Arch. Biochem. Biophys. 207, 141-147 (1981)

23 Noell, W.K.: Possible mechanisms of photoreceptor damage by light in mammalian eyes. Vision Res. 20, 1163-1171 (1980)

24 Sporn, M.B., Squire, R.A., Brown, C.C., Smith, J.M., Wenk, M.L. and Springer, S.: 13-cisretinoic acid: Inhibition of bladder carcinogenesis in the rat. Science 195, 487-489 (1977)

25 Harisiadis, L., Miller, R.C., Hall, E.J. and Borek, C.: A vitamine $A$ analogue inhibits radiationinduced oncogenic transformation. Nature 274, 486-487 (1978)

26 Smith, G.J.: Reaction of retinol and retinal with singlet oxygen. Photochem. Photobiol. 38, 119120 (1983) 